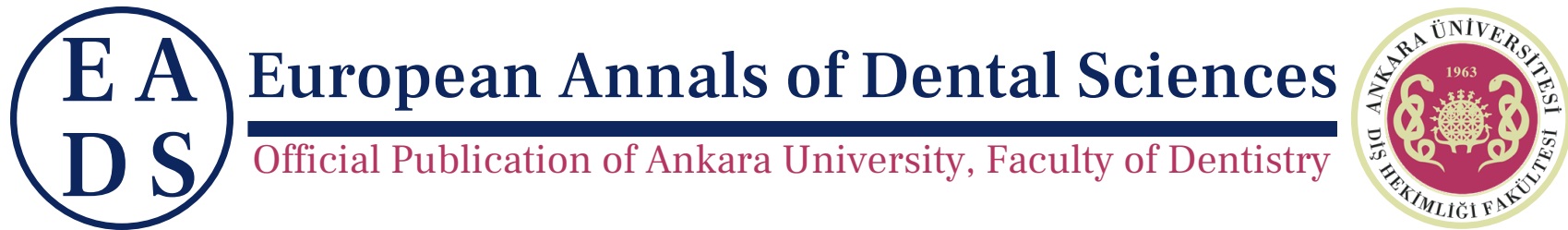

EADS, 2021, 48 (3), 124-128

e-ISSN : 2757-6744

Article Received : June, 22021

doi : 10.52037/eads.2021.0020

Article Accepted : August, 42021

\title{
Pre-eruptive Intra-coronal Resorption: A Report of Two Cases and Review of the Literature
}

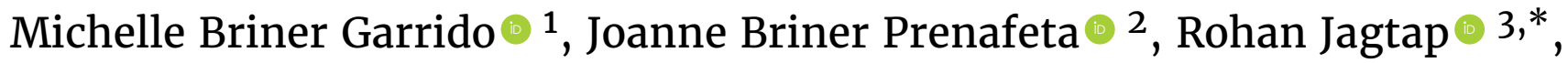 \\ Christopher D. Matesi $\odot 4$ and Andres Briner $\odot 2$
}

${ }^{1}$ DDS, University of Missouri-Kansas City School of Dentistry Department of Oral Pathology, Radiology, and Medicine, Kansas City, MO, USA and ${ }^{2}$ DDS, BRINMAX, Oral and Maxillofacial Imaging Center, Santiago, Chile and ${ }^{3}$ DDS, University of Mississippi Medical Center School of Dentistry Division of Oral and Maxillofacial Radiology Department of Care Planning and Restorative Sciences, MS, USA and 4DDS, University of Florida College of Dentistry Department of Oral and Maxillofacial Diagnostic Sciences, FL, USA

*Corresponding Author; drrohanjagtap@gmail.com

\begin{abstract}
Pre-eruptive intracoronary resorption is a rare condition mainly affecting pediatric patients. It is a radiographic finding in the dentin of the crown of an unerupted tooth just below the enamel-dentin junction. It is visualized in radiographs as a radiolucent coronal lesion of variable depth; it is well-defined and located in the dentin adjacent to the amelodentinal limit of an unerupted tooth. Historically, this lesion was misdiagnosed as caries and was misnamed pre-eruptive caries, when in reality they were pre-eruptive intracoronal resorptions. We present two rare cases of pre-eruptive intracoronary resorption in patients that needed radiographs for orthodontic purposes. The Oral \& Maxillofacial Radiologist detected the existence of pre-eruptive intracoronary resorption in mandibular second premolars and mandibular second molar. These radiographic findings provided the clinician with the advantage of knowing this condition before teeth eruption, allowing for conservative treatment and periodic radiographic follow-up.
\end{abstract}

Key words: intracoronal resorption; internal resoprtion; pre-eruptive intracoronal resorption

\section{Introduction}

The pre-eruptive intracoronal resorption (PEIR) is an incidental finding on routine dental radiographs. ${ }^{1}$ Typically, PEIR affects only one tooth, and whenever it affects more than one, it is usually its contralateral counterpart. However, multiple affected teeth have also been reported. In most cases, it only affects the external dentinal third while the enamel and the pulp remain intact. ${ }^{2}$ However, there have been cases in which the lesion is severe, affecting the enamel, pulp and in some cases, total resorption of the affected tooth have been reported (most of the cases are third molars). ${ }^{3,4}$ Nevertheless, these severe cases are very rare and do not represent the common course of PEIR.

Clinically, the affected teeth have no defects on the enamel surface and therefore there are no observable clinical differences with their contralateral counterparts. ${ }^{5,6}$ Patients are usually asymptomatic, although severe cases, which are the mi- nority, might be accompanied by pain. ${ }^{7}$ The main diagnostic method is radiographs, whereas the gold standard is histologic examination, which is not possible to do in all cases since it requires tooth extraction. ${ }^{5,8}$

Regarding the etiology and pathogenesis of this entity, no consensus has been reached. Several hypotheses have been proposed, however the most accepted hypothesis postulates that during tooth development there is damage to the reduced enamel epithelium, generating micro perforations through which resorptive cells from the dental follicle, surrounding bone and connective tissue invade the crown of the tooth in formation. These resorptive cells (osteoclasts, odontoclasts and macrophages) are then responsible for the resorption we see in PEIR. Additional factors that influence these cells are unknown. ${ }^{3-5,8-10}$

The prevalence of pre-eruptive intracoronary resorption is also a controversial issue and is varied in different clinical studies. This may be due to the fact that the apparent prevalence 
of this lesion may differ depending on the radiographic technique used and the age of the patient (it is more prevalent in mixed dentition). PEIR usually affects molars and premolars and rarely affects canines and incisors. ${ }^{3}$ Additionally, no association has been found between pre-eruptive intracoronary resorption and sex, race, medical conditions, systemic conditions or patients who have been previously treated with fluoride. ${ }^{2,6}$

In radiographic analysis, intraoral radiographs are typically the modality of choice for the detection of caries and coronal lesions. However, in these cases where we must observe lesions in non-erupted teeth, bite-wing and periapical radiographs could leave out vital information. Panoramic radiographs are a more appropriate technique than intraoral radiographs since it allows an overview of all teeth in formation and/or eruption, including third molars. Cone-Beam CT (CBCT) is not a radiographic technique that is requested routinely so often times the initial encounter with such lesions is via two dimensional radiography. That being said, follow up monitoring of these lesions should ideally be done with CBCT. ${ }^{2,5,10,11}$

Although the recommended treatment protocol is conservative management and monitoring, the unsupported enamel (due to reduced dentinal support) does have less resistance to masticatory forces. This, in some cases, may lead to tooth structure fracture and exposure of the dentin to the oral cavity which permits susceptibility to secondary caries invasion. In these cases, despite having opted initially for conservative treatment, ultimately a restorative treatment will be necessary. ${ }^{11-13}$

Likewise, if the lesion causes symptoms, affects more than $2 / 3$ of the dentin or has a progressive nature, more aggressive treatments may be necessary (i.e. surgical procedures on unerupted teeth with permanent or temporary filling). It should be reaffirmed that the symptomatic, progressive and severe cases are the minority for non-third molar teeth. The severe cases are more likely associated with third molars or have been described to be associated with hypomineralizations. There are other factors that dictate the course of treatment as well, such as the time it takes for the tooth to erupt, caries risk, and patient's follow-up compliance. ${ }^{11}$ The prognosis ultimately depends on the extent of the injury and its progress. ${ }^{2,3,7,8,11}$ This case report presents rare cases of the pre-eruptive intracoronal resorption in 11-year-old female and 10- year-old male that were identified as incidental findings on panoramic images.

\section{Case Report}

\section{Case 1}

An eleven year-old, female patient referred by his orthodontist presented to BRINMAX Oral and Maxillofacial Imaging Center, Santiago, Chile for a panoramic radiograph. Patient had no contributory medical history. On the panoramic image, well-defined radiolucent lesions were observed adjacent to the amelodentinal limit in its mesio-occlusal aspect in the mandibular second premolars bilaterally (Figure 1). Despite its radiographic similarity to dental caries, a carious process was ruled out given the teeth in question were unerupted and had no communication with the oral cavity. The differential diagnosis of pre-eruptive intracoronal resorption was made and the clinician was informed and educated at that time about the existence of these lesions. This allowed the dentist to conservatively treat these lesions in early stages preventing further complications. After eruption of both premolars, the dentist in charge observed that clinically the crown and enamel of these teeth were undamaged, and immediately after these teeth communicated with the oral cavity, sealants were applied to the grooves and fissures with the main objective of preventing carious lesions from occurring (Figure 2).

Follow-up bite-wing and periapical radiographic imaging was carried out 4 months later (radiographs not available) which showed no progression of the lesions, pulpal invasion, or secondary caries. The patient remained asymptomatic and there was no clinical evidence of caries or tooth structure fracture. The ideal in this case would be to perform periodic followups with a standardized radiographic technique that would allow to check if the lesions progress or stay static. If more detailed information is required, a СВCT would be indicated.

\section{Case 2}

A ten year-old male presented to BRINMAX Oral and Maxillofacial Imaging Center, Santiago, Chile. His treating doctor requested a CBCT for orthodontic purposes. When the report of this case was being done by the oral radiologist, a well-defined radiolucent lesion, located on the coronal dentin adjacent to the amelodentinal junction of mandibular second right molar was seen. After talking to the treating dentist, we corroborated that this tooth has not yet erupted into the oral cavity, letting us confirm this was a case of pre-eruptive intracoronal resorption (Figure 3). For this case, we requested previous radiographs of the patient to see if this entity was also seen on previous images. A less than a year older panoramic radiograph was sent to us (Figure 4) were we noticed that the same lesion can be seen, but not as clear as in the CBCT, making it easier to neglect. Unfortunately, we do not have follow-up images of this case since the lesion is asymptomatic and there are no current indications to order another radiograph.

Pre-eruptive intracoronary resorption is a radiographic finding. It can be seen in radiographs as well-defined radiolucent lesions of variable depth, located in the coronary dentin adjacent to the amelodentinal limit of an unerupted tooth. Severe cases have been described in the literature, where tooth pulp has been affected and tooth extraction had been the only possible treatment. However, in all cases in which we have diagnosed this entity, we have never witnessed a severe or progressive case, so that conservative treatment has been applied to all cases successfully. ${ }^{1,4}$

Panoramic, bite-wing and periapical radiographs are the most requested by dentists on a routine basis. These images are not the ideal modalities to be able to diagnose PEIR due the superimposition and distortion of structures, and in the case of the bite-wing and periapical, the insufficient field of view. There are cases where the resorption cannot be seen in two dimensional radiographs, and only can be visualized on CBCT, which is why the presence of these lesions have often been under appreciated (Figure 5). Yet, taking into consideration the pros and cons of conventional two dimensional radiography, cone beam computed tomography (CBCT) may ultimately provide the ideal evaluation for these lesions since it projects structures and allows evaluation of the lesion in all three planes of space. The CBCT is the exam to choose when it is necessary to make a more comprehensive evaluation of suspected PEIR. ${ }^{1-4,7,8,11}$

PEIR usually affects only one tooth, but it can be found in multiple teeth at once, as was described in this case. Bilateralism is a rare event and is barely described in the literature. Clinically, most of the affected teeth have no defects or lesions on the enamel surface and therefore there are no observable differences with their contralateral counterparts. ${ }^{1-3}$ This precludes the necessity of periodic radiographic monitoring. Early diagnosis is extremely important; once the tooth erupts; it has a high possibility of being invaded by rapidly progressing caries and may ultimately affect the dental pulp. $7,8,10,11,14$ In the fol- 


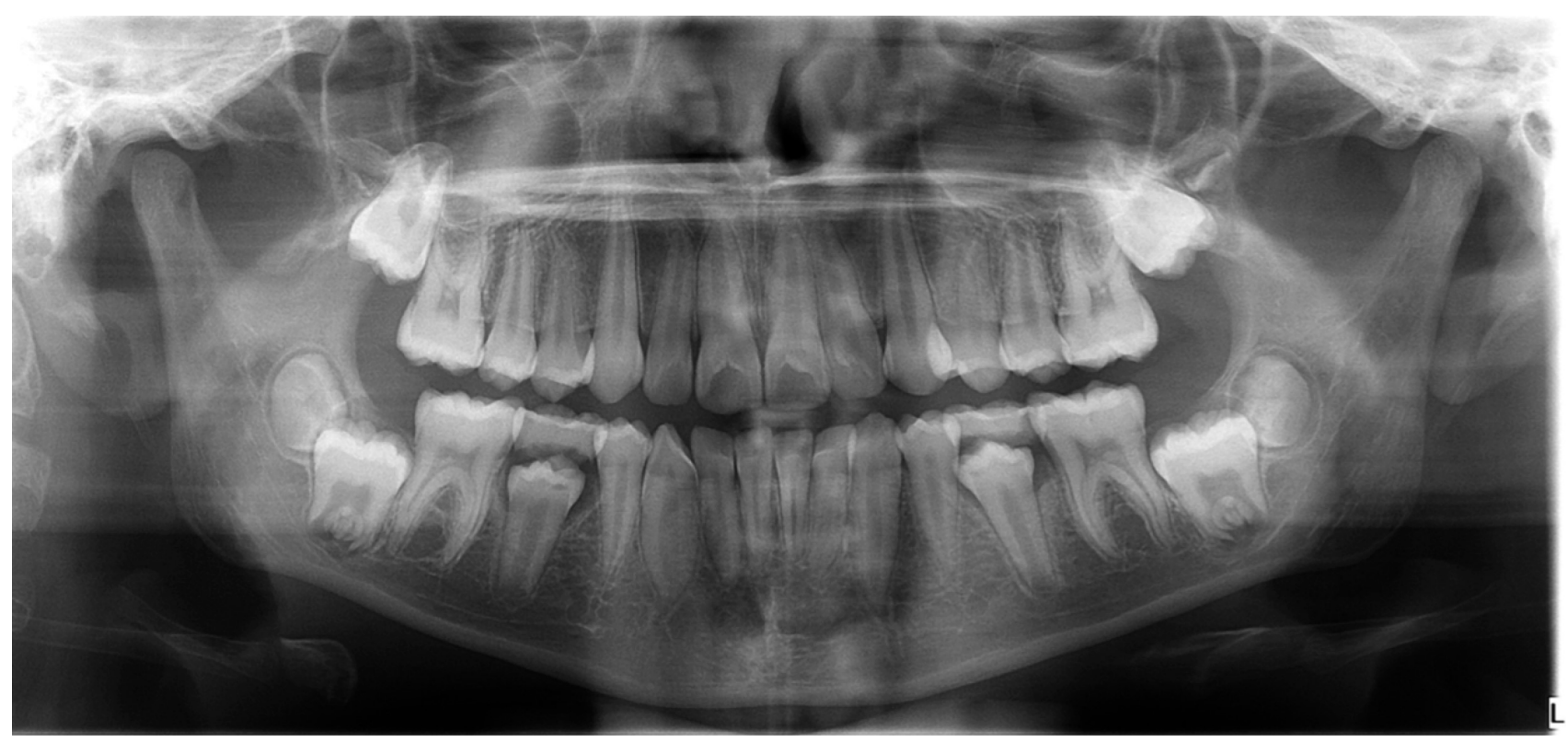

Figure 1. Panoramic radiograph of case 1 showing PEIR on the mandibular second premolars

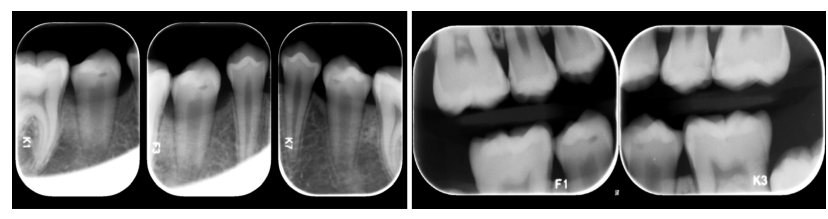

Figure 2. Case 1 periapical radiograph follow-up (after sealant placement)

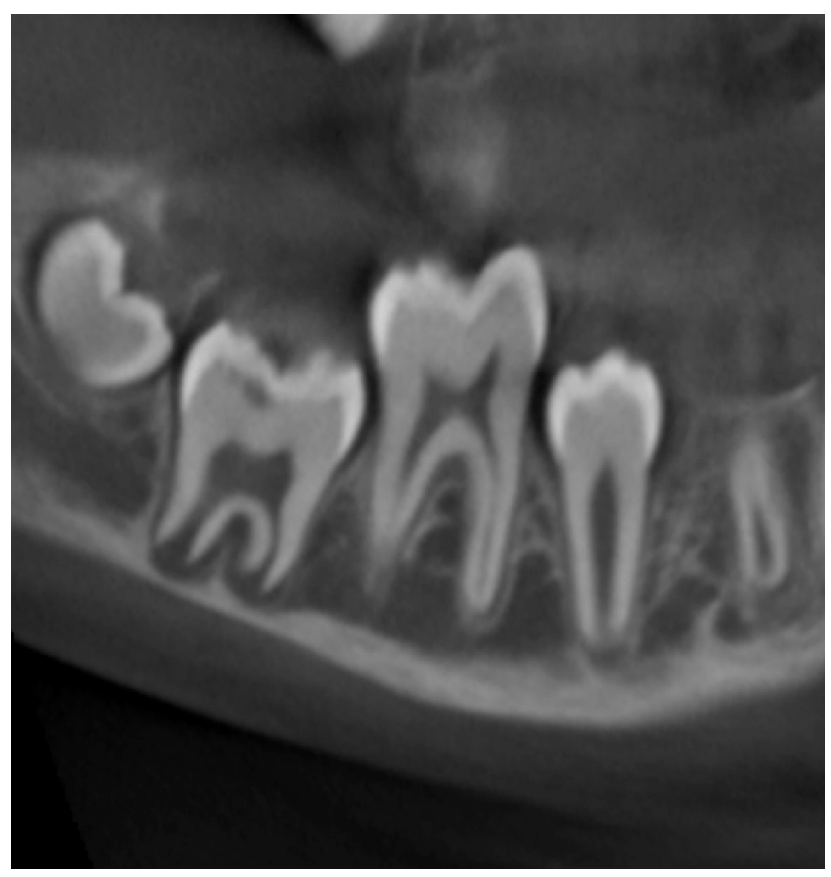

Figure 3. Reconstructed CBCT showing PEIR in the mandibular right second molar

lowing CBCT (Figure 6), there is an extensive carious lesion in a tooth with clinically unimpaired crown and enamel. This was misdiagnosed as occult caries, when in reality the correct diagnosis is caries secondary to pre-eruptive intracoronary resorption. An early correct diagnosis and conservative treatment may have prevented carious invasion and potential pulpal

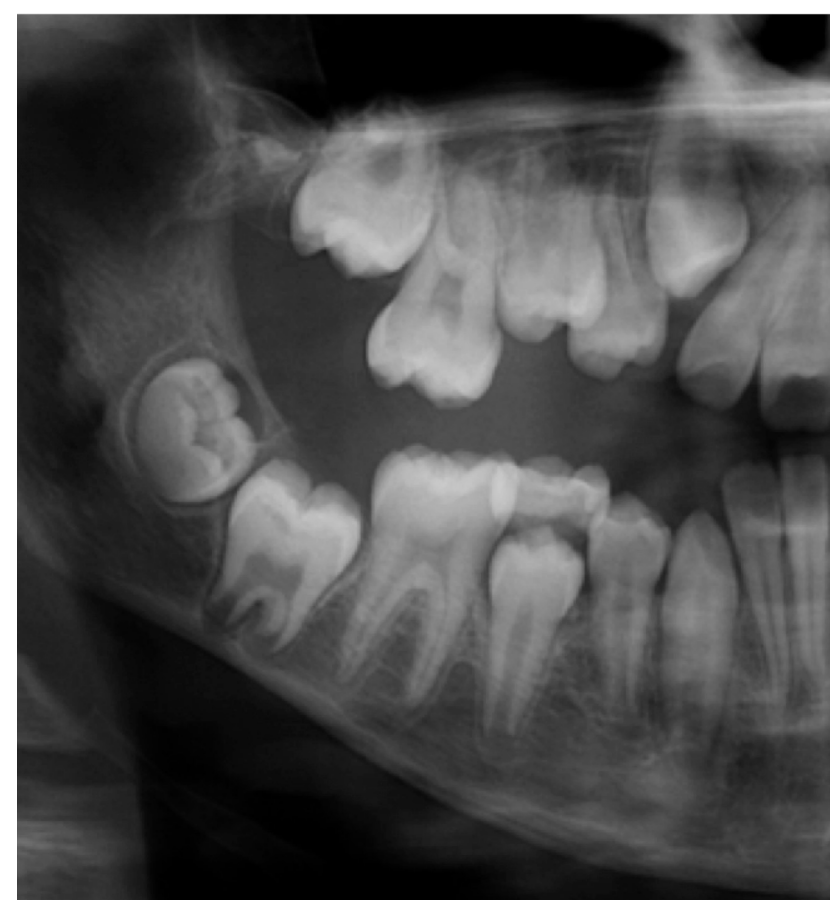

Figure 4. Previous panoramic showing PEIR in the mandibular second molar

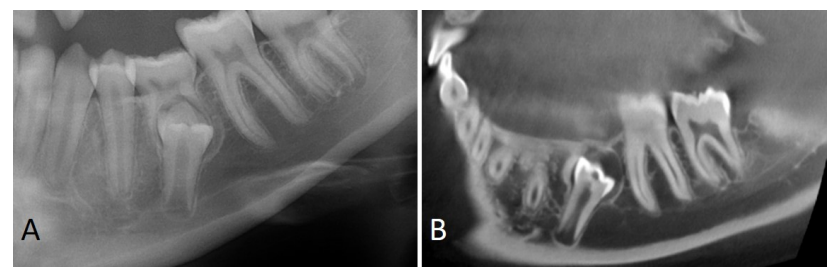

Figure 5. A mandibular second premolar with PEIR in a panoramic radiograph (image $\mathrm{A}$ ) versus on a CBCT (image $\mathrm{B}$ )

involvement. It is fundamental that radiologists and dentists have knowledge of the existence of this entity so that it can be detected in a timely manner. Conservative treatment with pe- 


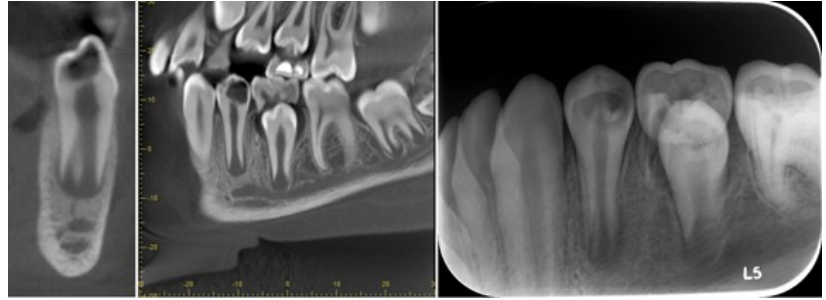

Figure 6. Caries on a previously affected inferior left first premolar with PEIR

riodic radiographic controls is the preferred treatment in these cases. The periodicity of these exams will depend on each case in particular taking into consideration the number of teeth involved, symptoms, extension of the lesion, progression, age of the patient and risk of caries. It is recommended that dentists and radiologists consider this entity when interpreting radiographs and emphasize the examination of unerupted teeth. It is fundamental to warn the clinician and the patient of the existence of this lesion since affected teeth, especially in people with a high caries risk, have a high probability of developing caries. It is important to note that a late diagnosis (after the total eruption of the tooth) can cause confusion, misdiagnosis and more commonly be confused with hidden caries, which has a completely different etiology and treatment. ${ }^{11,15}$

\section{Discussion}

The consensus in literature is that pre-eruptive intracoronary resorption has an idiopathic etiology. Future investigations are necessary to glimpse the etiology and pathogenesis PEIR. This may allow us to understand its nature and thus generate a better diagnosis and treatment plan. Most of the histologically studied cases have found that PEIR consist mainly of inflammatory resorptive cells with no evidence of microbial invasion, dental caries or pulpal degeneration. The cases in which the histological study have been carried out, have in common areas of degeneration of the dentinal tubules (which are later replaced by vascularized connective tissue), globular dystrophic calcifications, necrotic remnants of dentin and pre-collagen fibers. Cells such as osteoclasts, macrophages, odontoclasts, multinucleated giant cells and cells of chronic inflammation, adjacent to scalloped margins are also found in this lesion. ${ }^{5}$

Subsequently the hypothesis that it is a resorptive lesion by nature is accepted, although the triggers associated with the initiation of this resorptive process are still unknown. ${ }^{6,9,14}$ Given that the etiopathogenesis of this lesion is not yet clearly defined, consensus was reached that the etiology is idiopathic. $^{2}$

\section{Conclusion}

Pre-eruptive intracoronary resorption can be classified as static or progressive. In cases of non-progressive lesions, the scientific literature endorses a conservative treatment in which it is expected that the tooth erupts in its entirety and is treated with sealants, application of fluoride, dietary advice and oral hygiene instruction. Given that practically all cases are asymptomatic, it is recommended to monitor the lesion in time through radiographs and in the event that there is symptomatology or progression, immediate treatment is recommended to avoid progression of the lesion into the pulp tissue. ${ }^{9}$

\section{Author Contributions}

Joanne Briner Prenafeta and Andres Briner treated the patient; Christopher D. Matesi and Michelle Briner Garrido collected and analyzed the data; Rohan Jagtap and Michelle Briner Garrido led the writing.

\section{Conflict of Interest}

Author Michelle Briner Garrido, Author Joanne Briner Prenafeta, Author Rohan Jagtap, Author Christopher D. Matesi, Author Andres Briner declare that they have no conflict of interest.

\section{Authors' ORCID(s)}

$\begin{array}{ll}\text { M.B.G. } & 0000-0002-0737-9848 \\ \text { J.B.P. } & 0000-0001-7265-5434 \\ \text { R.J. } & 0000-0002-9115-7235 \\ \text { C.D.M. } & 0000-0001-7972-0585 \\ \text { A.B. } & 0000-0001-5079-4708\end{array}$

\section{References}

1. Al-Batayneh OB, AlJamal GA, AlTawashi EK. Pre-eruptive intracoronal dentine radiolucencies in the permanent dentition of Jordanian children. Eur Arch Paediatr Dent. 2014;15(4):229-36. doi:10.1007/s40368-013-0104-x.

2. Barra SG, Villalobos MIDO, Penido CVDSR, Recchioni ACB, Visconti MAPG. Pre-interruptive intracoronal resorption: clinical and radiographic follow-up. RGO-Revista Gaúcha de Odontologia. 2017;65:282-285.

3. Cossellu G, Angiero F, Farronato G. Complete pre-eruptive idiopathic crown resorption. Pediatr Dent. 2014;36(7):14750.

4. Wong L, Khan S. Occult caries or pre-eruptive intracoronal resorption? A chance finding on a radiograph. Pediatr Dent. 2014;36(5):429-32.

5. Hemani K, Deepa G. Pre eruptive intracoronal resorptiona review. International Journal of Current Advanced Research. 2017;6:3589-3591.

6. Manmontri C, Mahasantipiya PM, Chompu-Inwai P. Preeruptive Intracoronal Radiolucencies: Detection and Nine Years Monitoring with a Series of Dental Radiographs. Case Rep Dent. 2017;2017:6261407. doi:10.1155/2017/6261407.

7. Brunet-Llobet L, Lahor-Soler E, Miranda-Rius J. Oral pain due to severe pre-eruptive intracoronal resorption in permanent tooth. Eur J Paediatr Dent. 2014;15(3):332-4.

8. Lenzi R, Marceliano-Alves MF, Alves F, Pires FR, Fidel S. Pre-eruptive intracoronal resorption in a third upper molar: clinical, tomographic and histological analysis. Aust Dent J. 2017;62(2):223-227. doi:10.1111/adj.12444.

9. Moskovitz M, Holan G. Pre-eruptive intracoronal radiolucent defect: a case of a nonprogressive lesion. J Dent Child (Chic). 2004;71(2):175-8.

10. Umansky M, Tickotsky N, Friedlander-Barenboim S, Faibis S, Moskovitz M. Age Related Prevalence of Pre-Eruptive Intracoronal Radiolucent Defects in the Permanent Dentition. J Clin Pediatr Dent. 2016;40(2):103-6. doi:10.17796/10534628-40.2.103.

11. Manmontri C, Chompu-Inwai P, Mahasantipiya PM, Prapayasatok S. Prevalence of pre-eruptive intracoronal radiolucencies in Thai children and adolescents: A retrospective study. J Investig Clin Dent. 2018;9(2):e12303. doi:10.1111/jicd.12303. 
12. Spierer WA, Fuks AB. Pre-eruptive intra-coronal resorption: controversies and treatment options. J Clin Pediatr Dent. 2014;38(4):326-8. doi:10.17796/jcpd.38.4.dm7652634h12705v.

13. Uzun I, Gunduz K, Canitezer G, Avsever H, Orhan K. A retrospective analysis of prevalence and characteristics of pre-eruptive intracoronal resorption in unerupted teeth of the permanent dentition: a multicentre study. Int Endod J.
2015;48(11):1069-76. doi:10.1111/iej.12404.

14. Gera A, Zilberman U. Diagnosis and Management of Hidden Caries in a Primary Molar Tooth. Int J Clin Pediatr Dent. 2017;10(1):99-102. doi:10.5005/jp-journals-10005-1415.

15. Chouchene F, Hammami W, Ghedira A, Masmoudi F, Baaziz $A$, Fethi $M$, et al. Treatment of pre-eruptive intracoronal resorption: A scoping review. Eur J Paediatr Dent. 2020;21(3):227-234. doi:10.23804/ejpd.2020.21.03.13. 